\title{
Longitudinal Plasma Kallikrein Levels and Their Association With the Risk of Cardiovascular Disease Outcomes in Type 1 Diabetes in DCCT/EDIC
}

\author{
Miran A. Jaffa, ${ }^{1}$ Ionut Bebu, ${ }^{2}$ Deirdre Luttrell,,3 Barbara H. Braffett, ${ }^{2}$ John M. Lachin, ${ }^{2}$ Kelly Hunt, ${ }^{4}$ \\ Maria Lopes-Virella, ${ }^{3}$ Louis Luttrell, ${ }^{3}$ Timothy J. Lyons, ${ }^{3}$ Ayad A. Jaffa, ${ }^{3,5}$ and the DCCT/EDIC \\ Research Group*
}

Diabetes 2020;69:2440-2445 | https://doi.org/10.2337/db20-0427

\begin{abstract}
We determined the relationship between plasma kallikrein and cardiovascular disease (CVD) outcomes as well as major adverse cardiovascular events (MACE) in the Diabetes Control and Complications Trial (DCCT)/ Epidemiology of Diabetes Interventions and Complications (EDIC) cohort of type 1 diabetes (T1D). Plasma kallikrein levels were measured longitudinally in 693 subjects at DCCT baseline (1983-1989), midpoint (1988-1991), and end (1993) and at EDIC years 4-6 (1997-1999), 8-10 (2001-2003), and 11-13 (2004-2006). Cox proportional hazards regression models assessed the association between plasma kallikrein levels and the risk of CVD. In unadjusted models, higher plasma kallikrein levels were associated with higher risk of any CVD during DCCT/EDIC (hazard ratio [HR] $=1.16$ per $20 \mathrm{nmol} / \mathrm{L}$ higher levels of plasma kallikrein; $P=0.0177$ ) as well as over the EDIConly period (HR $=1.22 ; P=0.0024)$. The association between plasma kallikrein levels and the risk of any CVD remained significant during the EDIC follow-up after adjustment for age and mean $\mathrm{HbA}_{1 \mathrm{c}}(\mathrm{HR}=1.20 ; P=0.0082)$ and in the fully adjusted model for other CVD risk factors $(H R=1.17 ; P=0.0330)$. For MACE, higher plasma kallikrein levels were associated with higher risk in the unadjusted ( $H R=1.25 ; P=0.0145)$, minimally adjusted $(\mathrm{HR}=1.23 ; P=0.0417$, and fully adjusted $(\mathrm{HR}=1.27 ; P=$ 0.0328 ) models for EDIC only. These novel findings indicate that plasma kallikrein level associates with the risk of any CVD and MACE in T1D individuals.
\end{abstract}

Inflammatory mediators, cytokines, and growth factors play important roles in the development of atherosclerosis, the leading cause of death in patients with diabetes and a major risk factor for cardiovascular disease (CVD) events $(1,2)$. Similarly, the presence of diabetes confers a two- to fourfold greater risk of any cardiovascular event compared with individuals without diabetes $(3,4)$. The maladaptive risk factors and signals that promote the initiation and progression of diabetic vascular disease remain incompletely defined.

Plasma kallikrein is linked to the activation of the intrinsic coagulation pathway resulting in thrombus formation and inflammation (5-7). Higher plasma kallikrein activity is associated with higher blood pressure and increased albumin excretion rate in patients with type 1 diabetes (T1D) (8). Moreover, we have recently shown that circulating levels of plasma kallikrein are associated with carotid intima-media thickness and its progression in subjects with T1D (9). These data suggest that plasma kallikrein may have substantial value both as a pathogenic risk marker of inflammation-induced tissue injury and as a therapeutic target. Herein, we performed longitudinal measurements of plasma kallikrein in a large, well-characterized cohort of participants from the Diabetes Control and Complications Trial (DCCT)/Epidemiology of Diabetes Interventions and Complications (EDIC) study to evaluate

\footnotetext{
1Epidemiology and Population Health Department, Faculty of Health Sciences, American University of Beirut, Beirut, Lebanon

${ }^{2}$ The Biostatistics Center, The George Washington University, Rockville, MD ${ }^{3}$ Department of Medicine, Medical University of South Carolina, Charleston, SC ${ }^{4}$ Department of Public Health Sciences, Medical University of South Carolina, Charleston, SC

5Department of Biochemistry and Molecular Genetics, Faculty of Medicine, American University of Beirut, Beirut, Lebanon

Corresponding author: Ayad A. Jaffa, aj24@aub.edu.lb

Received 25 April 2020 and accepted 11 August 2020
}

Clinical trial reg. nos. NCT00360893, NCT00360815, clinicaltrials.gov

This article contains supplementary material online at https://doi.org/10.2337/ figshare.12801350

*A complete list of investigators and members of the DCCT/EDIC Research Group appears in N Engl J Med 2017;376:1507-1516.

(C) 2020 by the American Diabetes Association. Readers may use this article as long as the work is properly cited, the use is educational and not for profit, and the work is not altered. More information is available at https://www.diabetesjournals .org/content/license. 
whether antecedent plasma kallikrein levels are associated with or predict future CVD events.

\section{RESEARCH DESIGN AND METHODS}

\section{Subjects}

The DCCT enrolled a total of 1,441 subjects who were randomly assigned to receive either intensive therapy $(n=$ 711) aimed at lowering glycemic values as close to the nondiabetic range as possible or conventional therapy $(n=$ 730) aimed at maintaining clinical well-being with no specific glucose targets $(10,11)$. After an average of 6.5 years of follow-up, the DCCT ended in 1993, and all participants were encouraged to adapt intensive therapy and were referred back to their health care providers. One year later, the EDIC follow-up observational study enrolled $98 \%$ of the surviving DCCT cohort, with $94 \%$ of the surviving DCCT participants still actively participating after an additional 20 years of follow-up.

This study was conducted on a subset of 693 subjects representative of the full DCCT/EDIC cohort (Supplementary Table 1). The DCCT and EDIC studies were approved by the institutional review boards of all participating DCCT/EDIC centers and all participants provided written informed consent.

\section{CVD Risk Factors}

DCCT/EDIC visits consist of a detailed medical history including demographic and behavioral risk factors, medical outcomes, and a physical examination that includes measurements of height, weight, sitting blood pressure, and pulse rate $(10,11)$. Blood samples collected at each visit were assayed centrally for $\mathrm{HbA}_{1 \mathrm{c}}$. Fasting lipids (triglycerides, total and HDL cholesterol) were measured annually during DCCT and in alternate years during EDIC and were evaluated centrally (11). Concurrent medication usage was collected during EDIC but not during the DCCT.

\section{Plasma Kallikrein Measurement}

Plasma kallikrein levels were measured by the hydrolysis of S-2302 (H-D-Prolyl-L-Phenylalanyl-L-arginine-p-nitroaniline dihydro-chloride; Chromogenix) at six time points collected at DCCT: baseline of DCCT, pass 1 (1983-1989); midpoint of DCCT, pass 2 (1988-1991); closeout of DCCT, pass 3 (1993); EDIC years 4-6, pass 4 (1997-1999); EDIC years 8-10, pass 5 (2001-2003); and EDIC years 11-13, pass 6 (2004-2006). All six time points for each subject were run on the same plate using the same reagent stocks. This is a robust assay with inter- and intra-assay coefficients of variance of $3 \%$ and $8 \%$, respectively. In addition, each sample was run in duplicate wells, and each run included identical plate controls (pooled plasma) to ensure plate-to-plate comparability. Plates that did not meet quality control standards were reanalyzed.

\section{Cardiovascular Outcomes}

CVD events were ascertained based on medical history, electrocardiogram, and available medical records, and they were adjudicated by a committee masked to DCCT treatment group and $\mathrm{HbA}_{1 \mathrm{c}}$. The primary CVD outcome ("any CVD") was defined as the time to the first occurrence of CVD death, nonfatal myocardial infarction (MI), nonfatal stroke, subclinical MI on electrocardiogram, angina confirmed by ischemic changes with exercise tolerance testing or by clinically significant obstruction on coronary angiography, revascularization (with angioplasty or coronary artery bypass), or congestive heart failure $(12,13)$. The secondary CVD outcome, major adverse cardiovascular events (MACE) included only the time to cardiovascular death, nonfatal MI, or nonfatal stroke, whichever occurred first $(14,15)$. Participants free of a CVD event were administratively censored as of 31 December 2013.

\section{Statistical Analyses}

Summary statistics of the DCCT baseline characteristics were used to assess whether the 693 participants with available plasma kallikrein measurements included in these analyses were representative of the entire DCCT/ EDIC cohort $(n=1,441)$. Participants with DCCT baseline plasma kallikrein values below and above the median value were compared using the Wilcoxon rank-sum test for continuous variables and the $\chi^{2}$ test for binary variables.

Longitudinal generalized estimating equation models using an unstructured correlation matrix and a class variable for visit (i.e., pass) assessed the association between risk factors and repeated measures of plasma kallikrein over time.

Separate Cox proportional hazards regression models assessed the association between plasma kallikrein values and the risk of any CVD and MACE. Models were unadjusted (model 1); minimally adjusted by age and $\mathrm{HbA}_{1 c}$ (model 2); and fully adjusted by age, $\mathrm{HbA}_{1 \mathrm{c}}$, duration of diabetes, HDL, total cholesterol, BMI, log triglycerides, systolic blood pressure (SBP), sex, smoking, menopause, and use of statins and lipid-lowering medications (model 3 ). With the exception of age, duration of diabetes, and sex, all variables (including the plasma kallikrein values) were included as time-dependent covariates. $\mathrm{HbA}_{1 c}, \mathrm{HDL}$, total cholesterol, BMI, triglycerides, and SBP were defined using updated mean values, with weights proportional to the time between visits.

Given that plasma kallikrein values during EDIC were higher than those during DCCT and that most of the CVD events occurred during EDIC follow-up, both the generalized estimating equation and the Cox models were fitted over the entire DCCT/EDIC follow-up as well as only during EDIC. The EDIC-only time-to-event analyses excluded subjects with CVD events during DCCT, and time was reset to zero at the time of the first EDIC evaluation.

The longitudinal models employed complete data, while the time-to-event models carried forward plasma kallikrein values for time points with missing values. All analyses were performed using SAS (SAS Institute, Cary, NC) and $\mathrm{R}$, and $P$ values $\leq 0.05$ were considered nominally significant. 


\begin{tabular}{|c|c|c|c|c|}
\hline & All $(n=693)$ & $\begin{array}{c}\text { Plasma kallikrein } \\
<65.7 \mathrm{nmol} / \mathrm{L} \\
(n=344)\end{array}$ & $\begin{array}{l}\text { Plasma kallikrein } \\
\geq 65.7 \mathrm{nmol} / \mathrm{L} \\
(n=345)\end{array}$ & $P$ value ${ }^{*}$ \\
\hline \multicolumn{5}{|l|}{ Characteristics } \\
\hline Treatment group (intensive) & $343(49)$ & $167(49)$ & $173(50)$ & 0.6748 \\
\hline Cohort (primary prevention) & 341 (49) & $174(51)$ & $163(47)$ & 0.3812 \\
\hline Current smoker & $115(17)$ & $65(19)$ & $49(14)$ & 0.0975 \\
\hline Age (years) & $26.9 \pm 6.9$ & $26.9 \pm 6.7$ & $26.8 \pm 7.0$ & 0.9616 \\
\hline Sex (male) & $372(54)$ & $223(65)$ & $153(45)$ & $<0.0001$ \\
\hline BMI $\left(\mathrm{kg} / \mathrm{m}^{2}\right)$ & $23.3 \pm 2.7$ & $23.0 \pm 2.7$ & $23.5 \pm 2.7$ & 0.0088 \\
\hline Duration of diabetes (months) & $65.8 \pm 48.2$ & $63.5 \pm 46.8$ & $68.5 \pm 49.5$ & 0.2542 \\
\hline $\mathrm{HbA}_{1 \mathrm{c}}(\%)$ & $9.0 \pm 1.6$ & $8.7 \pm 1.4$ & $9.3 \pm 1.7$ & $<0.0001$ \\
\hline $\mathrm{HbA}_{1 \mathrm{c}}(\mathrm{mmol} / \mathrm{mol})$ & $75 \pm 17$ & $72 \pm 15$ & $78 \pm 19$ & $<0.0001$ \\
\hline $\mathrm{SBP}(\mathrm{mmHg})$ & $114.8 \pm 11.2$ & $115.4 \pm 11.1$ & $114.3 \pm 11.2$ & 0.1493 \\
\hline HDL cholesterol (mg/dL) & $50.4 \pm 12.2$ & $49.2 \pm 11.3$ & $51.5 \pm 12.9$ & 0.0484 \\
\hline Total cholesterol (mg/dL) & $173.5 \pm 32.7$ & $168.2 \pm 32.4$ & $179.1 \pm 32.1$ & $<0.0001$ \\
\hline Triglycerides (mg/dL) & $79.6 \pm 46.3$ & $75.0 \pm 48.3$ & $84.2 \pm 43.9$ & 0.0005 \\
\hline \multicolumn{5}{|l|}{ CVD outcomes } \\
\hline Any CVD & $77(11)$ & $35(10)$ & $42(12)$ & \\
\hline MACE & $35(5)$ & $14(4)$ & $21(6)$ & \\
\hline
\end{tabular}

Data are $n(\%)$ or mean \pm SD, unless otherwise noted. $P$ values $\leq 0.05$ are presented in boldface type. ${ }^{*}$ Comparing the two plasma kallikrein groups.

\section{Data and Resource Availability}

Access to the DCCT/EDIC data are available through the National Institute of Diabetes and Digestive and Kidney Diseases Repository (https://repository.niddk.nih.gov/ studies/edic/).

\section{RESULTS}

Among the 693 participants at DCCT baseline, 54\% were males, and $49 \%$ were from the former DCCT intensive treatment group. Mean duration of diabetes was $5.5 \pm$ 4 years; mean age was $27 \pm 7$ years; and mean $\mathrm{HbA}_{1 \mathrm{c}}$ was $9 \pm 1.6 \%$. Significant differences occurred in sex, BMI, $\mathrm{HbA}_{1 c}$, HDL, total cholesterol, and triglycerides between participants with plasma kallikrein values below and above the median $(65.7 \mathrm{nmol} / \mathrm{L})$. A total of $77 \mathrm{CVD}$ events (18 during DCCT and 59 during EDIC) and $35 \mathrm{MACE}$ (9 during DCCT and 27 during EDIC) were observed among the 693 participants in this study (Table 1).

The plasma kallikrein values had an intraclass correlation coefficient of 0.53 over the six time points, and the median values increased from $65.7,61.8$, and $63.3 \mathrm{nmol} / \mathrm{L}$ for the three time points during DCCT to 90.0, 91.5, and $92.9 \mathrm{nmol} / \mathrm{L}$ for the three time points during EDIC (Supplementary Table 2). This trend was consistent within sex and treatment group (data not shown). Table 2 also reports the number of CVD events within each interval (i.e., pass $1-2,2-3$, and so on).

On the basis of the magnitude of the $z$ score values in a multivariable longitudinal model including both DCCT and EDIC (all six time points) (Table 2), higher plasma kallikrein values were more strongly associated with higher mean BMI, $\mathrm{HbA}_{1 \mathrm{c}}$, total cholesterol, and with female sex, and less so with triglycerides. In a similar model including only the three EDIC time points, higher plasma kallikrein values were more strongly associated with higher mean triglycerides, $\mathrm{BMI}$, and $\mathrm{HbA}_{1 \mathrm{c}}$ and less so with higher mean HDL, smoking (higher in nonsmokers), and treatment group (higher in intensive) (Table 2).

Table 3 presents the Cox models for CVDs and MACE during the combined DCCT/EDIC follow-up as well as during the EDIC follow-up only. In the unadjusted CVD models, higher plasma kallikrein values were associated with a higher risk of CVD during DCCT/EDIC $(P=0.0177)$ as well as over the EDIC period alone $(P=0.0024)$. The association between plasma kallikrein values and the risk of CVD remained significant during the EDIC follow-up after adjustment for age and mean $\operatorname{HbA}_{1 c}(P=0.0082)$ and in the fully adjusted model $(P=0.0330)$, though it was not significant over the combined DCCT/EDIC study. For MACE, higher plasma kallikrein values were associated with a higher risk of CVD in unadjusted, minimally adjusted, and fully adjusted models during EDIC only $(P=0.0145$, 0.0417 , and 0.0328 , respectively) but not over the combined DCCT/EDIC follow-up (Table 3).

Additional analyses showed the associations between the DCCT baseline plasma kallikrein values and risk of any CVD and MACE over the combined DCCT/EDIC follow-up, which were qualitatively similar to the associations between the plasma kallikrein values using all six timepoints and the subsequent risk of any CVD and MACE (Supplementary Table 3).

\section{DISCUSSION}

In the current study, we demonstrate that plasma kallikrein levels are strongly related to sex, $\mathrm{HbA}_{1 c}$, BMI, total cholesterol, smoking, and total triglycerides and are 


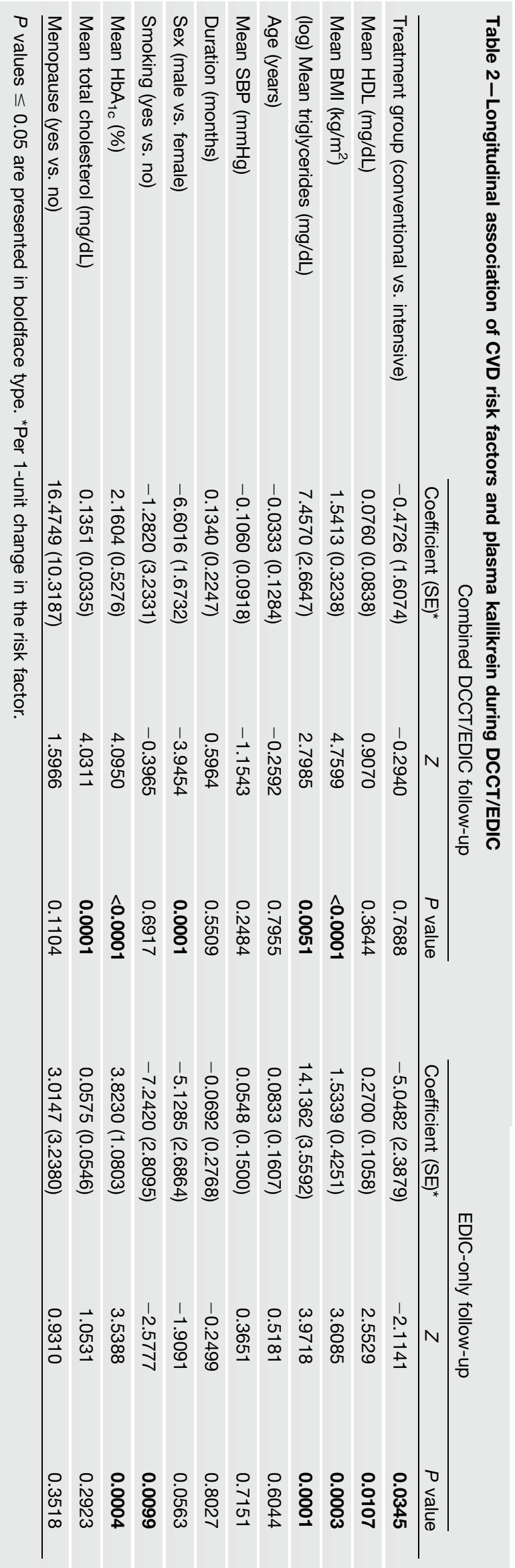


Table 3-HRs for any CVD and MACE per $20 \mathrm{nmol} / \mathrm{L}$ change of plasma kallikrein with no adjustment (model 1), minimal adjustment (model 2), and full adjustement (model 3)

\begin{tabular}{|c|c|c|c|c|c|c|}
\hline Outcome & Follow-up & Model $^{\star}$ & $H R^{\star \star}$ & $95 \% \mathrm{Cl}$ & $Z$ & $P$ value \\
\hline \multirow[t]{6}{*}{ Any CVD } & DCCT and EDIC & 1 & 1.1566 & $1.0263,1.3050$ & 2.3714 & 0.0177 \\
\hline & & 2 & 1.1005 & $0.9665,1.2520$ & 1.4451 & 0.1484 \\
\hline & & 3 & 1.0831 & $0.9474,1.2397$ & 1.1747 & 0.2401 \\
\hline & EDIC only & 1 & 1.2202 & $1.0745,1.3872$ & 3.0417 & 0.0024 \\
\hline & & 2 & 1.2034 & $1.0491,1.3791$ & 2.6445 & 0.0082 \\
\hline & & 3 & 1.1681 & $1.0121,1.3442$ & 2.1323 & 0.0330 \\
\hline \multirow[t]{6}{*}{ MACE } & DCCT and EDIC & 1 & 1.1474 & $0.9627,1.3656$ & 1.5382 & 0.1240 \\
\hline & & 2 & 1.0702 & $0.8813,1.2999$ & 0.6776 & 0.4980 \\
\hline & & 3 & 1.1226 & $0.9156,1.3764$ & 1.1197 & 0.2628 \\
\hline & EDIC only & 1 & 1.2520 & $1.0449,1.5006$ & 2.4453 & 0.0145 \\
\hline & & 2 & 1.2348 & $1.0080,1.5124$ & 2.0368 & 0.0417 \\
\hline & & 3 & 1.2694 & $1.0202,1.5820$ & 2.1346 & 0.0328 \\
\hline
\end{tabular}

$P$ values $\leq 0.05$ are presented in boldface type. *Model 1: plasma kallikrein; model 2: plasma kallikrein, age, and mean $\mathrm{HbA}$; $;$ model 3 : plasma kallikrein, age, mean $\mathrm{HbA}_{1 \mathrm{c}}$, duration of diabetes, mean $\mathrm{HDL}$, mean total cholesterol, mean BMI, (log) mean triglycerides, mean $\mathrm{SBP}$, sex, smoking, menopause, and use of statin and lipid-lowering medication. ${ }^{*} \mathrm{HRs}$ (and Cls) denote the hazard ratio and confidence interval corresponding to $20 \mathrm{nmol} / \mathrm{L}$ increase in the plasma kallikrein values.

associated with the risk of developing CVD events. The association between plasma kallikrein and the risk of CVD was strongest during the EDIC period when most of the CVD events occurred. In the fully adjusted model (model 3 ), a test of interaction between plasma kallikrein and a binary variable defined as 1 for EDIC follow-up and 0 for DCCT follow-up confirmed an interaction for both any $\operatorname{CVD}(P=0.0160)$ and MACE $(P=0.0120)$. The ability of plasma kallikrein to associate or predict CVD outcomes remained after controlling for established CVD risk factors including age, sex, mean $\mathrm{HbA}_{1 c}$, duration of diabetes, mean SBP, smoking, mean HDL, mean total cholesterol, mean BMI, (log) mean triglycerides, menopause, and use of statins (lipid-lowering medication).

The data described herein support the current hypothesis that plasma kallikrein is a pathogenic risk determinant acting along a causal pathway for the development of CVD. Activation of plasma kallikrein leads to cleavage of kininogen to release the proinflammatory vasoactive peptide bradykinin, which, in turn, initiates a multitude of cellular signals that influence vascular and renal function $(5,16-19)$. We have also shown that plasma kallikrein can signal independently of bradykinin generation via activation of protease-activated receptors 1 and 2 to stimulate the release of proinflammatory cytokines and proliferation of vascular cells through transactivation of epidermal growth factor receptors (20). Plasma kallikrein levels were associated with the risk of CVD and stroke in men, and individuals who experienced an MI had higher levels of plasma kallikrein $(21,22)$. Children with abnormal lipid profiles and metabolic syndrome had elevated plasma kallikrein levels that were associated with CVD risk factors (23). Furthermore, polymorphisms in plasma kallikrein (rs3733402) that results in reduced plasma kallikrein activity are associated with reduced CVD risk (24). In contrast, in the Tromso study, higher plasma kallikrein values were associated with protection against MI (25), likely due to differences in the populations studied, age, and metabolic state.

Strengths of our study include the outstanding follow-up with $94 \%$ of the surviving cohort still actively participating, the standardized assessment of longitudinal risk factors, including plasma kallikrein levels, and the adjudicated CVD outcomes. For event time outcomes, the power to detect associations with risk factors increases as the effect sizes (i.e., hazard ratios $[\mathrm{HRs}]$ ), and the number of events increase. The relatively small number of CVD events in our study (77 any CVD events and 35 MACE) may have limited our power to detect associations between the plasma kallikrein values and the risk of CVD over the combined DCCT/ EDIC follow-up. However, the consistent association between plasma kallikrein levels and the risk of both any CVD and MACE in the unadjusted and adjusted models during the EDIC follow up (when most of the events occurred) is reassuring. In particular, the HRs for MACE were slightly higher than the HRs for any CVD.

In summary, this study demonstrates for the first time that high levels of plasma kallikrein associate with the risk of developing CVD events in subjects with T1D. Unraveling the mechanisms responsible for the reported relationship between plasma kallikrein and CVD may identify novel targets for therapeutic interventions.

Funding. This work was supported by National Institutes of Health National Heart, Lung, and Blood Institute grants HL-077192, HL-087986 (A.A.J.), and HL-055782 (M.L.-V.). The DCCT/EDIC has been supported by cooperative agreement grants (1982-1993, 2012-2017, 2017-2022), and contracts (1982-2012) with the Division of Diabetes Endocrinology and Metabolic Diseases of the National Institute of Diabetes and Digestive and Kidney Diseases (current grant numbers U01-DK-094176 and U01-DK-094157), and through support by the National Eye Institute, the National Institute of Neurological Disorders and Stroke, the General Clinical Research Centers Program (1993-2007), and Clinical Translational 
Science Center Program (2006 to present), Bethesda, MD. Industry contributors provided free or discounted supplies or equipment to support participants' adherence to the study: Abbott Diabetes Care (Alameda, CA), Animas (West Chester, PA), Bayer Diabetes Care (North America Headquarters, Tarrytown, NY), Becton Dickinson (Franklin Lakes, NJ), Eli Lilly (Indianapolis, IN), Extend Nutrition (St. Louis, M0), Insulet Corporation (Bedford, MA), LifeScan (Milpitas, CA), Medtronic Diabetes (Minneapolis, MN), Nipro Home Diagnostics (Fort Lauderdale, FL), Nova Diabetes Care (Billerica, MA), Omron (Shelton, CT), Perrigo Diabetes Care (Allegan, MI), Roche Diabetes Care (Indianapolis, IN), and Sanofi (Bridgewater, NJ).

Industry contributors had no role in the DCCT/EDIC study.

Duality of Interest. No potential conflicts of interest relevant to this article were reported.

Author Contributions. M.A.J. developed the analytical plan and wrote, reviewed, and edited the manuscript. I.B. and J.M.L. developed the analytical plan; analyzed the data; and wrote, reviewed, and edited the manuscript. D.L. oversaw the measurements of plasma kallikrein. B.H.B., K.H., M.L.-V., L.L., and T.J.L. reviewed and edited the manuscript. A.A.J. conceived the study; amassed the resulting data; and wrote, reviewed, and edited the manuscript. A.A.J. is the guarantor of this work and, as such, had full access to all the data in the study and takes responsibility for the integrity of the data and the accuracy of the data analysis.

\section{References}

1. Moriya J. Critical roles of inflammation in atherosclerosis. J Cardiol 2019;73: 22-27

2. Ridker PM, Everett BM, Thuren T, et al.; CANTOS Trial Group. Antiinflammatory therapy with canakinumab for atherosclerotic disease. N Engl J Med 2017;377:1119-1131

3. Khunti K, Davies M, Majeed A, Thorsted BL, Wolden ML, Paul SK. Hypoglycemia and risk of cardiovascular disease and all-cause mortality in insulintreated people with type 1 and type 2 diabetes: a cohort study. Diabetes Care 2015;38:316-322

4. Secrest AM, Becker DJ, Kelsey SF, Laporte RE, Orchard TJ. Cause-specific mortality trends in a large population-based cohort with long-standing childhoodonset type 1 diabetes. Diabetes 2010;59:3216-3222

5. Björkqvist J, Jämsä A, Renné T. Plasma kallikrein: the bradykinin-producing enzyme. Thromb Haemost 2013;110:399-407

6. Weidmann H, Heikaus L, Long AT, Naudin C, Schlüter H, Renné T. The plasma contact system, a protease cascade at the nexus of inflammation, coagulation and immunity. Biochim Biophys Acta Mol Cell Res 2017;1864:2118-2127

7. Revenko AS, Gao D, Crosby JR, et al. Selective depletion of plasma prekallikrein or coagulation factor XII inhibits thrombosis in mice without increased risk of bleeding. Blood 2011;118:5302-5311

8. Jaffa AA, Durazo-Arvizu R, Zheng D, et al.; DCCT/EDIC Study Group. Plasma prekallikrein: a risk marker for hypertension and nephropathy in type 1 diabetes. Diabetes 2003;52:1215-1221

9. Jaffa MA, Luttrell D, Schmaier AH, et al.; DCCT/EDIC Research Group. Plasma prekallikrein is associated with carotid intima-media thickness in type 1 diabetes. Diabetes 2016;65:498-502

10. Epidemiology of Diabetes Interventions and Complications (EDIC) Research Group. Epidemiology of Diabetes Interventions and Complications (EDIC). Design, implementation, and preliminary results of a long-term follow-up of the Diabetes Control and Complications Trial cohort. Diabetes Care 1999;22: 99-111

11. The DCCT Research Group. The Diabetes Control and Complications Trial (DCCT). Design and methodologic considerations for the feasibility phase. Diabetes 1986;35:530-545

12. The DCCT Research Group. Lipid and lipoprotein levels in patients with IDDM diabetes control and complication. Trial experience. Diabetes Care 1992;15:886894

13. Lachin JM, Orchard TJ, Nathan DM; DCCT/EDIC Research Group. Update on cardiovascular outcomes at 30 years of the diabetes control and complications trial/epidemiology of diabetes interventions and complications study. Diabetes Care 2014;37:39-43

14. Bebu I, Braffett BH, Orchard TJ, Lorenzi GM, Lachin JM; DCCT/EDIC Research Group. Mediation of the effect of glycemia on the risk of CVD outcomes in type 1 diabetes: the DCCT/EDIC study. Diabetes Care 2019;42:1284-1289

15. Writing Group for the DCCT/EDIC Research Group. Coprogression of cardiovascular risk factors in type 1 diabetes during 30 years of follow-up in the DCCT/EDIC study. Diabetes Care 2016;39:1621-1630

16. Terzuoli E, Meini S, Cucchi $P$, et al. Antagonism of bradykinin B2 receptor prevents inflammatory responses in human endothelial cells by quenching the NF-kB pathway activation. PLoS One 2014;9:e84358

17. Tan $Y$, Wang B, Keum JS, Jaffa AA. Mechanisms through which bradykinin promotes glomerular injury in diabetes. Am J Physiol Renal Physiol 2005;288: F483-F492

18. Dhamrait SS, Payne JR, Li P, et al. Variation in bradykinin receptor genes increases the cardiovascular risk associated with hypertension. Eur Heart J 2003; 24:1672-1680

19. Nunes RAB, Lima LB, Tanaka NI, da Costa Pereira A, Krieger JE, Mansur AJ. Genetic associations of bradykinin type 2 receptor, alpha-adrenoceptors and endothelial nitric oxide synthase with blood pressure and left ventricular mass in outpatients without overt heart disease. Int J Cardiol Heart Vasc 2018;21: 45-49

20. Abdallah RT, Keum JS, El-Shewy HM, et al. Plasma kallikrein promotes epidermal growth factor receptor transactivation and signaling in vascular smooth muscle through direct activation of protease-activated receptors [published correction appears in J Biol Chem 2011;286:23620]. J Biol Chem 2010;285: 35206-35215

21. Merlo C, Wuillemin WA, Redondo M, et al. Elevated levels of plasma prekallikrein, high molecular weight kininogen and factor $\mathrm{XI}$ in coronary heart disease. Atherosclerosis 2002;161:261-267

22. Govers-Riemslag JWP, Smid M, Cooper JA, et al. The plasma kallikrein-kinin system and risk of cardiovascular disease in men. J Thromb Haemost 2007;5: 1896-1903

23. MacKenzie JA, Roosa KA, Gump BB, Dumas AK, Bendinskas KG. Plasma prekallikrein levels are positively associated with circulating lipid levels and the metabolic syndrome in children. Appl Physiol Nutr Metab 2010;35:518-525

24. Gittleman HR, Merkulova A, Alhalabi 0 , et al. A cross-sectional study of KLKB1 and PRCP polymorphisms in patient samples with cardiovascular disease. Front Med (Lausanne) 2016;3:17

25. Wilsgaard T, Mathiesen EB, Patwardhan A, et al. Clinically significant novel biomarkers for prediction of first ever myocardial infarction: the Troms $\emptyset$ Study. Circ Cardiovasc Genet 2015;8:363-371 